\section{S29 MANNOSE BINDING LECTIN DEFICIENCY IN CHILDREN WITH RESPIRATORY INFECTION}

doi:10.1136/thx.2010.150912.29

${ }^{1} \mathrm{~S}$ Prudon, ${ }^{2} \mathrm{~J}$ Wake, ${ }^{2} \mathrm{D}$ Barge, ${ }^{1} \mathrm{C}$ Simmister, ${ }^{1} \mathrm{~S}$ Tremble, ${ }^{1} \mathrm{~V}$ Stevenson, ${ }^{1} \mathrm{~T}$ Flood, ${ }^{1} \mathrm{D}$ A Spencer. 'Great North Children's Hospital, Newcastle Upon Tyne, UK; ${ }^{2}$ Royal Victoria Infirmary, Newcastle Upon Tyne, UK

Introduction Mannose binding lectin (MBL) plays a crucial part in innate immunity by activating the complement pathway. MBL deficiency is common, it has been associated with pulmonary disease, but low levels may also be found in otherwise normal individuals. Little is known about the importance of MBL deficiency as a risk factor for recurrent upper and lower respiratory tract infections. ${ }^{12}$ We describe our experience of measuring MBL levels in children with recurrent upper and lower respiratory tract infections. Methods MBL levels and clinical data were extracted from immunology laboratory records, of children being investigated for acute or chronic recurrent respiratory tract infections between November 2008 and February 2010. MBL deficiency was defined as a level $<75 \mathrm{ng} / \mathrm{ml}$.

Results 489 children had serum MBL measured during this time period. 199 had recurrent respiratory infections and of those 36 were positive for MBL deficiency (Abstract S29 Figure 1). Deficiency was found in $29 \%$ of positive boys and $14 \%$ of positive girls. $20 \%$ of positive patients were $<2$ years, $16 \% 2-5$ years and $28 \%>5$ years at the time of investigation.

Conclusions $\mathrm{MBL}$ deficiency is common in our population. The true significance of this abnormality as a risk factor for respiratory infection in children remains to be determined. Case-control studies will be required to evaluate the relative importance of this abnormality in different subgroups of patients.
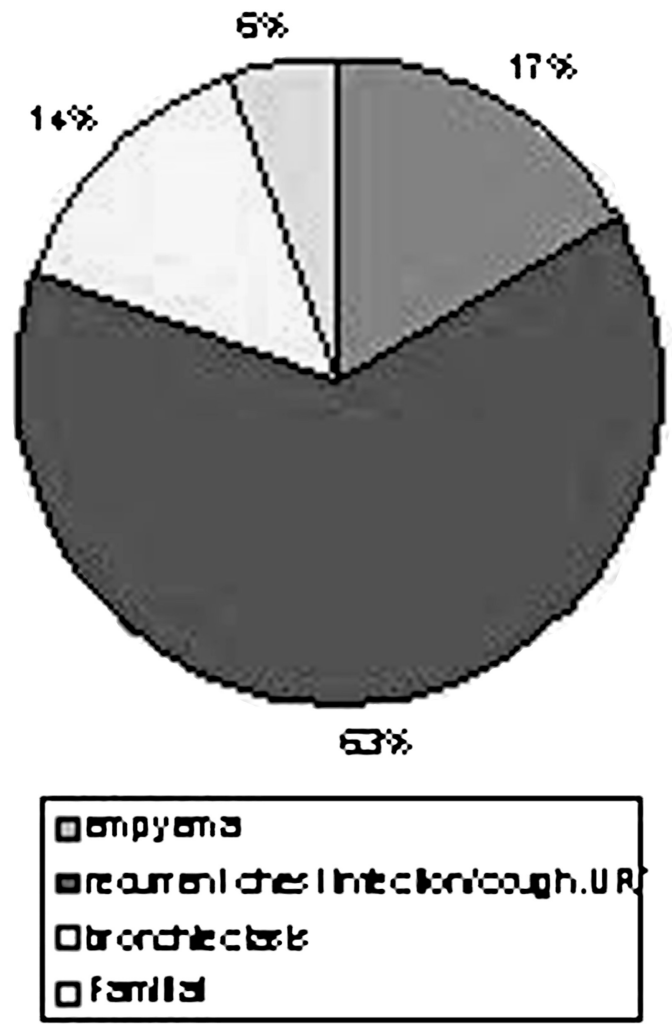

Abstract S29 Figure 1 The distribution of clinical problems of MBL deficient children.

\section{REFERENCES}

1. Aittoniemi J, Baer M, Soppi E, et al. Mannan binding lectin deficiency and concomitant immunodefects. Arch Dis Child 1998:78:245-8.

2. Brodlie M, Flood TJ, Spencer DA. Heterogeneity in the clinical manifestations of mannose binding lectin deficiency. J Pediatrics 2009;155:764-5.

\section{Linking airways inflammation and remodelling S30 TRUSS IS A REGULATOR OF TNF $\alpha-T N F-R 1$ INDUCED NF-KB ACTIVATION}

doi:10.1136/thx.2010.150912.30

${ }^{1} \mathrm{I} \mathrm{P}$ Horan, ${ }^{1} \mathrm{~A} \mathrm{~J}$ Langton, ${ }^{2} \mathrm{~S}$ Farrow, ${ }^{3} \mathrm{D} \mathrm{W}$ Riches, ${ }^{1} \mathrm{E} \mathrm{R}$ Chilvers, ${ }^{1} \mathrm{H}$ Parfrey. ${ }^{1}$ University of Cambridge, Cambridge, UK; ${ }^{2}$ Respiratory and Inflammation Centre GlaxoSmithKline, Stevenage, UK; ${ }^{3}$ National Jewish Medical and Research Center, Denver, USA

Introduction $\mathrm{TNF} \alpha$ is a pleiotropic cytokine that can exert opposing biological actions, either pro-inflammatory or pro-apoptotic through interaction with its cognate receptor TNF-R1. How this balance is regulated remains to be elucidated. One possible regulator is the novel TNF-R1 interacting protein TRUSS (TNF-R1 ubiquitous signalling and scaffold protein). Ectopic expression of TRUSS activates the transcription factors NF- $\kappa \mathrm{B}$ and AP-1.

Aims To determine the physiological role of TRUSS in TNF $\alpha$-TNFR1 signal transduction.

Methods Human epithelial (A549) cells were transfected with human TRUSS siRNA and responses to TNF $\alpha$ stimulation were assessed by RT-PCR, ELISA, immunoblotting and confocal microscopy.

Results TRUSS knockdown impaired secretion of the inflammatory chemokines IL- 6 and IL- 8 following prolonged TNF $\alpha$ stimulation. The maximal reduction of IL- $8 \mathrm{mRNA}$ and protein occurred after $12 \mathrm{~h}$ of TNF $\alpha$ incubation in the TRUSS deficient cells $(\mathrm{p}<0.03)$ whereas the IL- 6 responses were decreased after $24 \mathrm{~h} \quad(p<0.03)$ (Abstract S30 Figure 1). Furthermore, these effects were abrogated by cycloheximide or the NF-кB inhibitor Bay11-7085, indicating that the inflammatory chemokines were newly synthesised in response to $\mathrm{TNF} \alpha$ stimulation via an $\mathrm{NF}-\kappa \mathrm{B}$ dependent pathway. The upstream signalling molecules TNFR1, TRADD, TRAF2 and RIP were unaffected by TRUSS deficiency. However, phosphorylation of the p50 precursor p105 was augmented in the cytosolic fraction of TRUSS knockdown cells whilst total p50 in the nuclear fraction was reduced by $\mathrm{TNF} \alpha$ stimulation. This was associated with impaired nuclear translocation of the activated NF- $\kappa B$ subunit p65 and enhanced JNK phosphorylation.
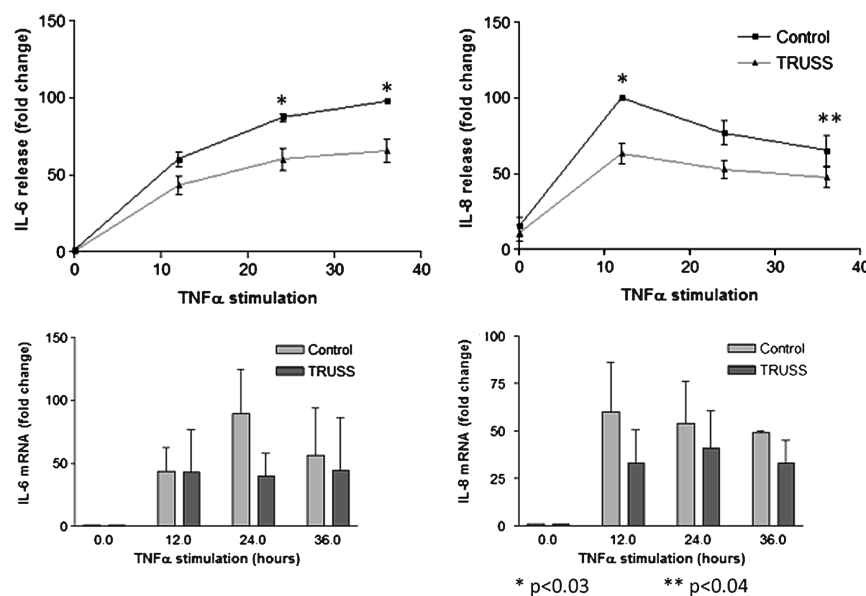

Abstract S30 Figure 1

Conclusions Our data suggest TRUSS is integral to TNF $\alpha$-TNF-R1 mediated NF- $\kappa B$ activation. We propose that TRUSS functions as a 
scaffold protein involved in the proteasomal processing of p105 into the p50 subunit. Inhibition of this process impairs the nuclear translocation of NF- $\kappa \mathrm{B}$ and the consequent $\mathrm{p} 65 / \mathrm{p} 50$ regulated gene transcription. Hence, TRUSS may be a novel target for modulating the inflammatory functions of TNF $\alpha$-TNF-R1 signalling.

\section{S31 CHARACTERISATION OF CELL ADHESION MOLECULE-1 IN LUNG MAST CELLS}

doi:10.1136/thx.2010.150912.31

E P Moiseeva, M Leyland, P Bradding. University of Leicester, Leicester, UK

Introduction and Objectives Cell adhesion molecule 1 (CADM1) is implicated in several diseases and a prognostic factor for lung cancer. In human lung mast cells (HLMC) it contributes to cell-cell adhesion and proliferation. Functional CADM1 isoforms arise from alternative splicing between exons 7 and 11. Exon 9 may be functionally important because it encodes a specific cleavage site for TNF-alpha-cutting enzyme, TACE, in the longest SP1 and SP6 isoforms. Our aim was to characterise the isoforms of CADM1 expressed in HLMC.

Methods CADM1 expression in isolated HLMC and human mast cell lines (HMC-1 and LAD2), was investigated using RT-PCR, cloning, and transfection.

Results Multiple highly glycosylated CADM1 isoforms were found in HLMCs and cell lines. The SP4 isoform containing exon 8 represented $\sim 80 \%$ of clones in both HLMCs and 'differentiated' LAD2 cells, and $\sim 96 \%$ in non-differentiated HMC-1 cells. The SP1 isoform with exons $8+9$ represented $\leq 20 \%$ of clones in HLMCs and LAD2 cells. A novel SP6 isoform with exons $8+9+10$ was found only in HLMCs ( $<5 \%$ of clones). More sensitive PCR analysis detected all these isoforms and an additional isoform SP3, lacking exons 8-10, in HLMCs and in cell lines. In contrast to these functional isoforms, non-functional isoforms were also found with two cryptic exons between exons 1 and 2, which cause a translational frame shift and premature termination of the protein. The cryptic exon B is located within the hot spot for SNPs. When the isoforms SP4, SP1 and SP6 were expressed in the HMC-1 cell line and the epithelial cell line HEK293 as fusion CADM1-GFP proteins, they were found at the cell periphery as well as in the cytoplasm. They were concentrated in cell boundaries in clumps of HMC1 cells.

Conclusions Multiple and novel CADM1 isoforms are found in HLMCs. The longest SP1 and SP6 isoforms might be involved in the negative regulation of HLMC adhesion following secretion of TACE after activation. Some cryptic exons are likely to be a result of specific SNPs, creating new branching points for splicing. Nonfunctional cryptic CADM1 isoforms may reduce mature protein expression and affect the function of CADM1 expressing cells.

\section{S32 CYCLICAL MECHANICAL STRETCH ENHANCES THE PRO- FIBROTIC RESPONSES OF PRIMARY EMBRYONIC FOETAL FIBROBLASTS, BUT NOT ADAM33 EXPRESSION}

doi:10.1136/thx.2010.150912.32

Antonio Noto, Wiparat Manuyakorn, Hans M Haitchi, Fabio Bucchieri, Donna Davies. The Brooke Laboratories, Division of Infection, Inflammation and Immunity, University of Southampton School of Medicine, Southampton, UK

Rationale A Disintegrin And Metalloprotease (ADAM) 33 is a susceptibility gene associated with asthma, bronchial hyperresponsiveness (BHR) and reduced lung function in young children. It is selectively expressed in mesenchymal cells, including bronchial smooth muscle and fibroblasts. We have previously shown that $A D A M 33$ expression increases during lung development when spontaneous peristaltic contractions of the airways commence. Therefore we hypothesised that mechanical strain induces $A D A M 33$ expression and affects smooth muscle/myofibroblast differentiation.

Methods Primary human embryonic fibroblasts from the pseudoglandular stage of lung development were cultured on flexible collagen-coated membranes and exposed to cyclical mechanical stretch (30\% amplitude, 12 cycles per minute) for 48, 96 and $168 \mathrm{~h}$. Control cells were cultured on the same membranes without mechanical stretch. Quantitative RT-PCR was performed for $A D A M 33$ and a-smooth muscle actin (a-SMA), a marker of smooth muscle and myofibroblast differentiation. We also measured collagen III and IL-8 mRNA. Soluble collagen protein levels in culture supernatants were measured using soluble collagen assay.

Results $A D A M 33$ and a-SMA mRNA expression were not significantly affected by mechanical strain. In contrast, collagen III mRNA expression was increased fourfold by cyclical mechanical strain and there was a threefold increase in soluble collagen proteins in culture supernatants of stretched cells. Unexpectedly, IL-8 expression was also increased by cyclical mechanical strain.

Conclusion Mechanical strain did not appear to influence markers of smooth muscle differentiation. However, the increase in ECM production may indicate a requirement for stiffening of the airways as the tubular structures develop. We postulate that IL-8 is not proinflammatory in the context of airway development, and may be a paracrine growth factor for developing epithelial cells.

\section{S33 SUPPRESSION OF CONSTITUTIVE AND STIMULATED SECRETION OF HISTAMINE FROM HUMAN LUNG MAST CELLS BY A SECRETED FACTOR FROM LUNG EPITHELIAL CELLS}

doi:10.1136/thx.2010.150912.33

N Martin, G K Arthur, W Y H Wan, L Woodman, C E Brightling, I D Pavord, P Bradding Institute for Lung Health, Glenfield Hospital, Leicester, UK

Introduction Constitutive and IgE-dependent secretion of histamine from human lung mast cells (HLMC) is suppressed by direct contact with the BEAS-2B bronchial epithelial cell line, but not conditioned epithelial cell media. This suggests that direct contact or a low concentration/labile secreted factor is involved. We explored this relationship further using a Transwell co-culture system of HLMC with BEAS-2B monolayers or air liquid interface primary bronchial epithelial cultures (ALI) derived from asthmatic and healthy subjects.

Methods ALI and BEAS-2B were grown on Transwell membranes. IgE-sensitised HLMC were then cultured i) on the luminal surface of a BEAS-2B monolayer, ii) separated from the BEAS-2B basal surface by a Transwell membrane, or iii) on the well bottom with BEAS-2B and ALI sitting on the Transwell insert. Cells were cocultured for $16 \mathrm{~h}$, media removed, and HLMC stimulated with antiIgE for $30 \mathrm{~min}$. Parallel controls without epithelial cells were performed. Histamine concentrations were determined by radioenzymic assay.

Results $B E A S-2 B \quad(n=3)$ : Compared to no epithelium control, constitutive histamine secretion from HLMC in direct contact with BEAS-2B was suppressed by a mean ( \pm SEM) $57 \pm 15 \%(p=0.04)$, and by $55 \pm 9 \%$ when separated by Transwells $(p=0.02)$. IgEdependent secretion was suppressed by $79 \pm 8 \% \quad(p=0.04)$ from HLMC in direct contact with BEAS- $2 B$ and by $88 \pm 7 \%(p=0.03)$ when separated by Transwells, compared to control. ALI-culture 